\title{
Innumerable Radioiodine Negative Cutaneous Metastases From Papillary Thyroid Cancer
}

\author{
Nishikant A. Damle ${ }^{a}$ c, Soundararajan Ramya ${ }^{a}$, Harmandeep Singh ${ }^{a}$, Prashant Durgapal ${ }^{b}$, \\ Chandrasekhar Bal ${ }^{\text {a }}$, Sandeep Mathur ${ }^{\mathrm{b}}$, Praveen Kumar ${ }^{\mathrm{a}}$
}

\begin{abstract}
Differentiated thyroid cancer (DTC) is the most common endocrine malignancy and papillary thyroid carcinoma (PTC) accounts for a majority of these. Distant metastases, especially to the lung and bones, occur in about $10 \%$ of patients, who can still survive for many years. We report here the case of a 32 year old female who presented with multiple bilateral cervical lymphadenopathy, diagnosed as metastatic PTC on cytology. She underwent total thyroidectomy and bilateral radical neck dissection followed by radioiodine therapy. Subsequently she developed pulmonary and bone metastases, and during the course of successive radioiodine doses, presented with multiple, painless cutaneous and subcutaneous nodules which proved to be metastatic on cytopathology and immunostaining. Radioiodine uptake was not seen in these lesions, suggesting dedifferentiation of follicular cells. This case emphasizes three clinically important issues-firstly, although extremely rare, cutaneous nodules can represent metastatic PTC in patients with pre existing lung and bone lesions and portends a poorer prognosis. Secondly, these lesions may be dedifferentiated and hence not amenable to radioiodine therapy although the bone and lung lesions show radioiodine uptake. SPECT/CT helps us in determining if uptake of 131-I is in the skin lesions or underlying other sites. Redifferentiating agents may play a role in management of such disease. Lastly, immunostaining with CK19 and Thyroglobulin (Tg) can be highly useful to confirm the pathologic diagnosis.
\end{abstract}

Manuscript accepted for publication December 2, 2011

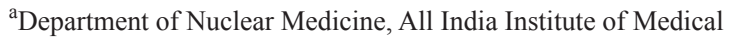
Sciences, New Delhi, India

${ }^{\mathrm{b}}$ Department of Pathology All India Institute of Medical Sciences, New Delhi, India

${ }^{\mathrm{c} C}$ Corresponding author: Nishikant A. Damle, Department of Nuclear Medicine, All India Institute of Medical Sciences, Ansari Nagar, New Delhi-110029, India. Email: nishikantdamle@rediffmail.com

doi: $10.4021 /$ jem $57 \mathrm{w}$
Keywords: Cutaneous metastases; Papillary thyroid cancer; Radioiodine; SPECT/CT

\section{Introduction}

Differentiated thyroid cancer (DTC) is the most common endocrine malignancy and papillary thyroid carcinoma (PTC) is the most common thyroid malignancy. It is usually associated with an excellent prognosis [1]. However, a small number of patients with PTC die of their disease. Distant metastases occur in about $10 \%$ of patients, who can still survive for many years. Most distant metastases appear in lung and bone. PTC is often multifocal and metastasizes to regional lymph nodes in about $40 \%$ of cases. Common sites of distant metastases from PTC are lung (49\%), bone (25\%), both lung and bone (15\%), and central nervous system (12\%) [2]. Other unusual sites of distant metastasis are the liver, kidneys and adrenal glands [3]. Follicular thyroid carcinoma (FTC) is the second most common type of thyroid carcinoma. FTCs account for approximately $10 \%$ to $15 \%$ of thyroid malignancies. Although FTCs are less frequently associated with lymph node metastases than PTC, patients with FTC may have distant metastases at presentation in up to $20 \%$ of cases [4].

Cutaneous metastases are a rare event, representing $0.7 \%$ to $2.0 \%$ of all cutaneous malignant neoplasms. They may be the first sign of a previously undiagnosed visceral malignancy or the initial presentation of a recurrent neoplasm. The frequency of cutaneous metastases according to the type of underlying malignancies varies with sex. In men, the most common internal malignancies leading to cutaneous metastases are lung cancer, colon cancer, melanoma, squamous cell carcinoma of the oral cavity, and renal cell carcinoma. In women, breast cancer, colon cancer, melanoma, lung cancer, and ovarian cancer are the most common malignancies leading to cutaneous metastasis [5].

Cutaneous metastasis from well differentiated thyroid carcinoma is rare. It usually occurs in the setting of disseminated neoplastic disease [3, 6-8]. Dahl et al reviewed pub- 


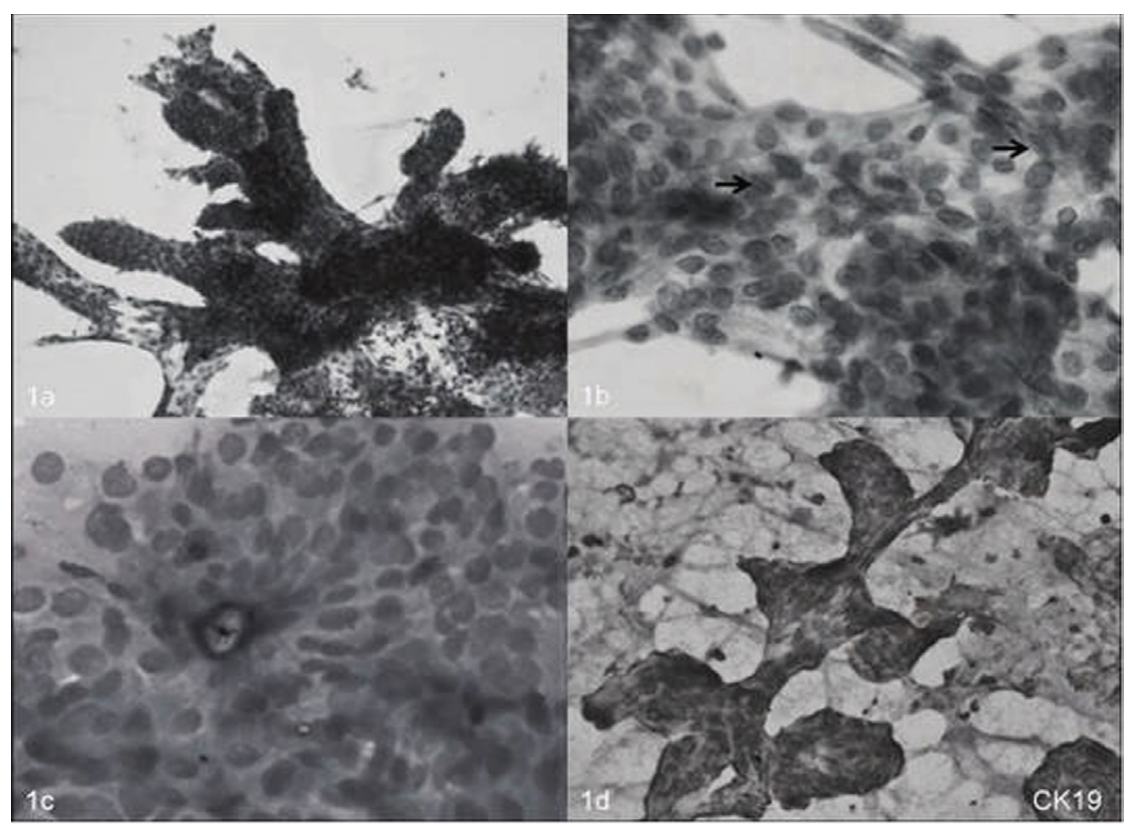

Figure 1. (a-d) Cytopathology revealing 1a) papillary structure 1b) nuclear grooves 1c) central calcification 1d) immunostaining positive for CK19.

lished literature (English) from 1964 onwards and found 43 cases of thyroid carcinoma with skin metastases. They found that papillary carcinoma was the most common thyroid cancer to result in skin metastases, representing $41 \%$ of cases, followed by follicular carcinoma at $28 \%$, with anaplastic carcinoma and medullary carcinoma each contributing $15 \%$ of cases. The scalp was the most common site of involvement [8]. In contrast, Koller et al reported that follicular carcinoma has a greater preponderance than papillary carcinoma for cutaneous metastases. The condition is equally as common in men and women [3]. Cutaneous metastases may be the initial manifestation of thyroid carcinoma [9]. Additionally,
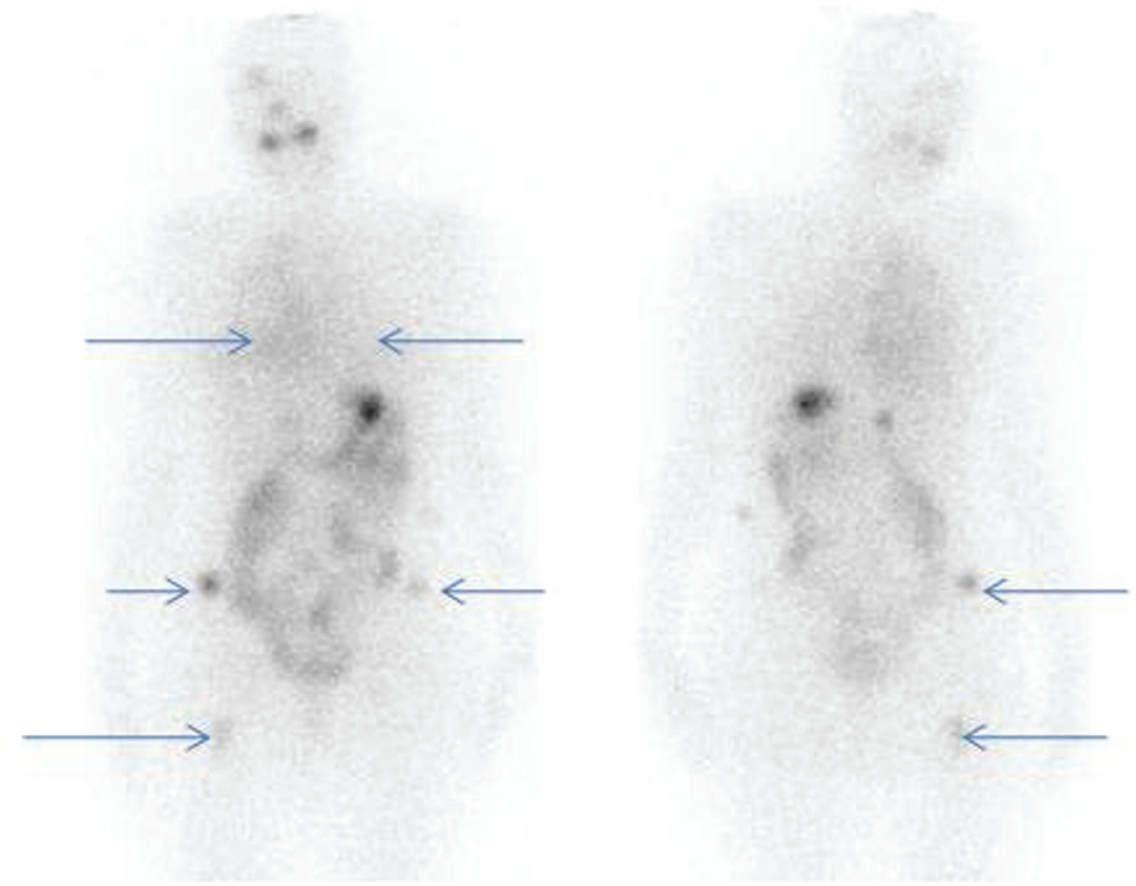

Figure 2. 131-I whole body post therapy scan (anterior and posterior images) showing radioiodine uptake in bilateral lung fields ( $R t>>L t$ ) and focal uptake in bilateral iliac bones and right femur. 

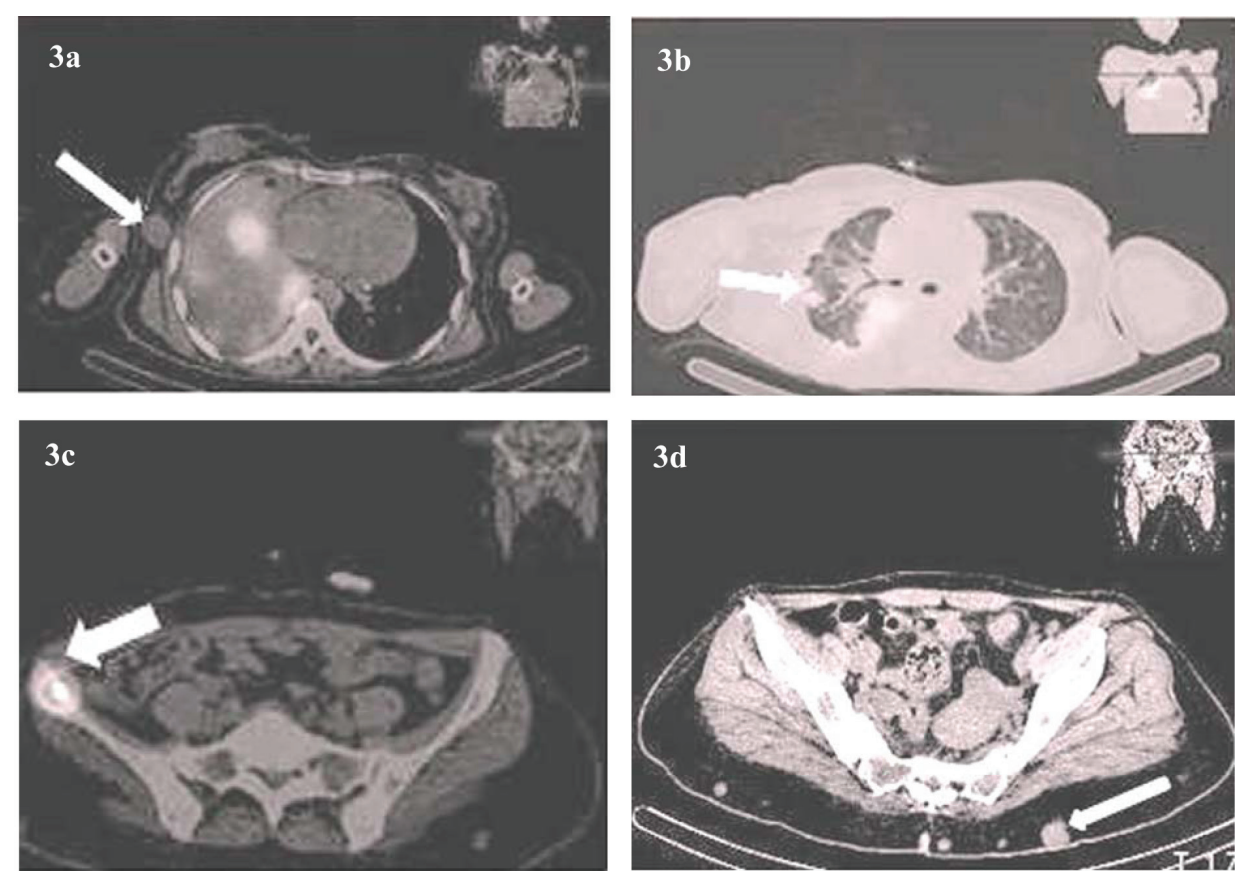

Figure 3. (a-d) Radioiodine SPECT/CT showing a) breast nodules b) lung metastases c) iliac bone metastasis d) multiple cutaneous and subcutaneous nodules.

cutaneous metastases can be the first evidence of recurrence of thyroid carcinoma [10].

In well differentiated thyroid carcinoma, cutaneous metastases indicate very poor prognosis [11]. In this report, we present a case of papillary carcinoma thyroid showing innumerable metastatic cutaneous and subcutaneous nodules.

\section{Case Report}

A 32 years old female presented to the surgeon with multiple cervical lymphadenopathy in 2001 and the cytopathological examination showed metastatic papillary carcinoma thyroid. She underwent total thyroidectomy and bilateral radical neck dissection. She was referred to the nuclear medicine department for radioiodine ablation only in 2004.131-I whole body scan (WBS) done with $2 \mathrm{mCi}$ of oral 131-I showed radioiodine uptake in remnant thyroid tissue, multiple bilateral cervical lymph nodes, bilateral lungs and left frontal and right parieto occipital regions. CT scan of brain showed metastatic lesions in left frontal, right parietal and right occipital lobes. As a next step she was given first therapy dose of 150 $\mathrm{mCi}$ radioiodine and the post therapy (PT) scan showed no additional lesions. As the brain lesions did not show radioiodine uptake, she underwent local radiotherapy for the same. Six months later she was given the second dose of $150 \mathrm{mCi}$ 131-I and the PT scan now showed radioiodine uptake only in bilateral lung fields.

She received further radioiodine at 6 monthly intervals. Her blood counts were checked routinely which were within normal limits. She completed 10 doses of radioiodine till 2010. Three months after the tenth dose, she presented with multiple painless subcutaneous nodules of variable size over the chest, back, forearm, abdomen, forehead, scalp and both legs. As she had known extensive metastatic disease a differential of cutaneous metastases from PTC was kept as the prime differential. She underwent an FNAC from a lesion in the left forearm which showed features compatible with metastasis from a carcinoma likely PTC. Moreover, immunostaining was positive for CK-19 and Thyroglobulin (Tg) confirming the diagnosis.

She received the next dose of radioiodine and the post therapy SPECT/CT showed increased radioiodine concentration in multiple pleural based nodules with right pleural effusion, bilateral lytic lesions in iliac bones, right femur and a focus in left frontoparietal region in the skull. However, none of the cutaneous lesions showed radioiodine uptake. The total dose given to her has now reached $1200 \mathrm{mCi}$. As radioiodine is the only treatment option in her case, she has been scheduled for the next therapy after six months.

\section{Discussion}

Cutaneous metastasis from well differentiated thyroid carcinoma is rare. It usually occurs in the setting of disseminated neoplastic disease [3, 6-8]. Literature reports 42 cases of cutaneous metastases, for which the primary thyroid tumor was PTC in 57\% and FTC in 42\% [3]. However, available literature has reported either solitary skin lesions or multiple 
localized skin nodules. To the best of our knowledge, a case of PTC presenting with innumerable cutaneous metastases all over the body has been rarely seen. The metastatic deposits usually present as flesh colored nodules that are tender, may be itchy. The lesions may be solitary or multiple and are almost always asymptomatic. Ulceration is not common. The presence of dermal metastases portends a poor prognosis, because visceral metastatic disease is almost invariably present. Thus, median survival after diagnosis of cutaneous metastases is about 19 months, because it usually occurs in the setting of disseminated neoplastic disease [8]. The cutaneous sites of metastasis appear to parallel the location and frequency of the most common sites of bony metastasis i.e. flat bones of the skull, pelvis, and sternum [12]. Our case also presented with painless multiple cutaneous nodules all over the body including scalp, breast, back and abdominal wall, and both upper and lower extremities. Metastatic thyroid carcinoma involving the skin can easily be mistaken for a primary adnexal skin tumor. The correct diagnosis requires a high index of suspicion and the liberal use of immunohistochemical stains. The development of antibodies against the thyroid transcription factor TTF-1 has provided a useful tool to screen for metastatic carcinomas. Immunohistochemical staining for thyroglobulin is usually helpful in making the diagnosis of metastatic thyroid carcinoma [10].

The above case indicates that the presence of new dermal lesions in a patient with a history of thyroid cancer should lead to a full examination of the skin for cutaneous nodules that may be metastatic and the diagnosis by cytology/biopsy and histological examination with thyroglobulin immunostaining. BRAFV600E mutation is present in a subset of cutaneous metastases from PTC [11]. The frequent identification of BRAFV600E mutation in patients with cutaneous metastases of PTC correlates with studies suggesting that BRAFV600E mutation may be associated with more aggressive disease [13]. These tumors need to be differentiated from clear cell hidradenoma, clear cell dermatofibroma, melanoma with clear cell change, and metastatic renal cell carcinoma. Pathologists need to be aware that cutaneous PTC metastases may also show this clear cell change and consider this entity in the differential diagnosis [11]. Although not a statistically significant difference, the prognosis is quite poor for patients with synchronous metastasis elsewhere at the time of cutaneous metastasis, in contrast to patients who have no disease elsewhere $(\mathrm{P}=0.07)$ [14].

Radioiodine therapy for iodine-concentrating welldifferentiated thyroid cancers including metastatic lesions, following surgery is of proven efficacy [15]. These patients, when appropriately treated, have a fairly good prognosis and can survive for many years. The physiological iodide uptake on the basolateral membrane of thyroid follicular cells is actively mediated by a membrane glycoprotein, the sodium iodide symporter (NIS). Because NIS forms the molecular basis for using radioiodine as scintigraphic imaging as a therapeutic agent for tissues with iodine uptake, NIS expression and function are critical in thyroid cancer management. Unfortunately, a loss of differentiation is observed in up to one-third of patients with differentiated thyroid cancer, paralleled by an increase in tumor grading and loss of iodine concentrating ability [16]. Previous reports of cutaneous metastases have also described features of increased risk including areas of dedifferentiation within the primary tumor and extrathyroidal extension $[8,10]$. In the case reported by us, none of the cutaneous nodules showed iodine concentration on SPECT/CT. This is an important advantage of this imaging modality as planar images of 131-I scans cannot discriminate between lesions overlapping in the antero-posterior plane. Iodine non-concentrating cutaneous nodules in this case revealed the aggressiveness of the disease manifesting as dedifferentiated metastasis. In such cases histopathological diagnosis remains the final way to establish the diagnosis. Moreover, immunostaining with CK19 and Tg is a valuable tool for confirming the diagnosis in cases where conventional microscopy is equivocal. Redifferentiation therapy with agents like retinoic acid or thalidomide may be of value in such patients.

Thus, although a rare entity, cutaneous metastasis from thyroid cancer should be kept as the first differential, when a patient of DTC, with already extensive metastatic disease presents with multiple cutaneous nodules. Occurrence of cutaneous lesions also explains the aggressive nature of the disease and portends a poor prognosis for the patient.

\section{Grant Support}

Dr. Nishikant A. Damle and other coauthors have no sources of funding to declare.

\section{Conflict of Interest}

Dr. Nishikant A. Damle and other coauthors have no conflict of interest pertatining to this manuscript.

\section{Authors' Contributions}

Nishikant A. Damle, Soundararajan Ramya, Harmandeep Singh, Praveen kumar and Chandrasekhar Bal conceptualized the report and reviewed the literature. Prashant Durgapal and Sandeep Mathur reviewed the pathology part of the report. All 7 authors were actively involved in managing the case.

\section{References}

1. LiVolsi VA, Albores-Saavedra J, Asa SL, et al. Papillary 
carcinoma.In: DeLellis RA, Lloyd RV, Heitz PU, Eng C, eds. World Health Organization Classification of Tumours Pathology \& Genetics of Tumours of Endocrine Organs. Lyon, France: IARC Press; 2004:57-66.

2. Shaha AR, Ferlito A, Rinaldo A. Distant metastases from thyroid and parathyroid cancer. ORL J Otorhinolaryngol Relat Spec. 2001;63(4):243-249.

3. Koller EA, Tourtelot JB, Pak HS, Cobb MW, Moad JC, Flynn EA. Papillary and follicular thyroid carcinoma metastatic to the skin: a case report and review of the literature. Thyroid. 1998;8(11):1045-1050.

4. Sobrinho Simoes M, Asa SL, Kroll TG, et al. Follicular carcinoma. In: DeLellis RA, Lloyd RV, Heitz PU, Eng C, eds. World Health Organization Classification of Tumours Pathology \& Genetics of Tumours of Endocrine Organs. Lyon, France: IARC Press; 2004:67-76.

5. Schwartz RA. Cutaneous metastatic disease. J Am Acad Dermatol. 1995;33(2 Pt 1):161-182; quiz 183-166.

6. Strate SM, Lee EL, Childers JH. Occult papillary carcinoma of the thyroid with distant metastases. Cancer. 1984;54(6):1093-1100.

7. Laskin WB, James LP. Occult papillary carcinoma of the thyroid with pulmonary metastases. Hum Pathol. 1983;14(1):83-85.

8. Dahl PR, Brodland DG, Goellner JR, Hay ID. Thyroid carcinoma metastatic to the skin: a cutaneous manifestation of a widely disseminated malignancy. J Am Acad Dermatol. 1997;36(4):531-537.

9. Pitlik S, Kitzes R, Ben-Bassat M, Rosenfeld JB. Thyroid carcinoma presenting as a solitary skin metastasis. $\mathrm{Cu}$ - tis. 1983;31(5):532-536.

10. Alwaheeb S, Ghazarian D, Boerner SL, Asa SL. Cutaneous manifestations of thyroid cancer: a report of four cases and review of the literature. J Clin Pathol. 2004;57(4):435-438.

11. Erickson LA, Jin L, Nakamura N, Bridges AG, Markovic SN, Lloyd RV. Clinicopathologic features and BRAF(V600E) mutation analysis in cutaneous metastases from well-differentiated thyroid carcinomas. Cancer. 2007;109(10):1965-1971.

12. Wolf BC, Sheahan K, DeCoste D, Variakojis D, Alpern HD, Haselow RE. Immunohistochemical analysis of small cell tumors of the thyroid gland: an Eastern Cooperative Oncology Group study. Hum Pathol. 1992;23(11):1252-1261.

13. Xing M, Westra WH, Tufano RP, Cohen Y, Rosenbaum E, Rhoden KJ, Carson KA, et al. BRAF mutation predicts a poorer clinical prognosis for papillary thyroid cancer. J Clin Endocrinol Metab. 2005;90(12):6373-6379.

14. Quinn TR, Duncan LM, Zembowicz A, Faquin WC. Cutaneous metastases of follicular thyroid carcinoma: a report of four cases and a review of the literature. Am J Dermatopathol. 2005;27(4):306-312.

15. Ward LS, Assumpcao LV. Thyroid cancer: prognostic factors and treatment. Arq Bras Endocrinol Metabol. 2004;48(1):126-136.

16. Schlumberger M, Lacroix L, Russo D, Filetti S, Bidart JM. Defects in iodide metabolism in thyroid cancer and implications for the follow-up and treatment of patients. Nat Clin Pract Endocrinol Metab. 2007;3(3):260-269. 